\title{
A Discrete Linear Stability Analysis of Two-dimensional Laminar Flow Past a Square Cylinder
}

\author{
NITIN KUMAR*, SACHIN TEJYAN, SUNIL CHAMOLI, PAWAN KUMAR PANT \\ Department of Mechanical Engineering, G. B. Pant Institute of Engineering and Technology, \\ Pauri, Uttarakhand, INDIA- 246194
}

\begin{abstract}
The present study focuses on the development of a numerical framework for predicting the onset of vortex sheading due to flow past a square cylinder. For this a discrete linear stability analysis framework for two-dimensional laminar flows have used. Initially the frame work is validating by using the analysis of thermal stability of flows in the discrete numerical sense. The two-dimensional base flow for various values of the controlling parameter (Reynolds number for flow past a square cylinder and Rayleigh number for doubleglazing problem) is computed numerically by using the lattice Boltzmann method. The governing equations, discretized using the finite-difference method in two-dimensions and are subsequently written in the form of perturbed equations with two-dimensional disturbances. These equations are linearized around the base flow and form a set of partial differential equations that govern the evolution of the perturbations. The eigenvalues, stability of the base flow and the points of bifurcations are determined using normal mode analysis. The eigenvalue spectrum predicts that the critical Reynolds number is 52 for the flow past a square cylinder. The results are consistent with the previous numerical and experimental observations.
\end{abstract}

Key-Words: - linear stability analysis; lattice Boltzmann; laminar flow; eigenvalue problem.

Received: September 30, 2020. Revised: April 28, 2021. Accepted: May 10, 2021. Published: May 27, 2021.

\section{Introduction}

The role of hydrodynamic stability in fluid mechanics that focuses on the evolution of small disturbances with time is of prime importance in understanding many natural phenomena, as well as in the analysis and design of many engineering systems. For example, it is pursued in civil aviation to design lifting surfaces with drag reduced by passive means. In these circumstances, keeping the flow stable over a wing to possibly as large an extent is the desired goal. The other application of stability is in the selection of kinematics governs the motion of self-propelled flapping wings with relevance to micro-air vehicles (MAVs) [1]. The fundamental ideas of linear stability analysis have been described in the classical works of Chandrasekhar [2] and Drazen [3]. The primary approach of most studies carried out on stability analysis to analytically calculate the critical value of flow governing parameter responsible for the hydrodynamic instability of simple fluid flow problems. Jackson [4] and Zebib [5] developed analytical tools to investigate a broad variety of nonparallel flow problems. The analytical model of stability analysis presented by Zebib [5] was modified and extended by Kim and Pearstein [6]. Fortin et.al. [7] used numerical approach with the linear and non-linear hydrodynamic instability of two-dimensional Poiseuille flows by reducing the problem to one-dimension using the OrrSommerfeld equation.

Clever and Busse [8] analytically examined the stability of two-dimensional Rayleigh-Benard convection and had shown that this steady flow undergoes a cross-roll instability at a low Rayleigh number $(R a=1708)$. The mechanism of the thermal convection instability of transition states was studied experimentally and theoretically by Busse [9]. Winters [10] presented an attractive threevariable model capturing many aspects of a twodimensional square air-filled cavity. Their numerical model suggested that the onset of the oscillatory convection occurs at a Hopf bifurcation in the steady-state equations for free convection in the Boussinesq approximation. Xin \& Le Quéré [11] investigated the stability of two-dimensional natural convection flows in a differentially heated square cavity with conducting horizontal walls for a large range of Prandtl numbers with respect to both twoand three-dimensional perturbations. 
It is clear that the analytical treatment of hydrodynamic instability is worth pursuing only for simple one-dimensional flow problems. A similar treatment for determining stability of non-parallel laminar fluid flows is inherently challenging and sometimes may not be even analytically feasible. In contrast, a numerical approach provides a more straightforward way to analyze non-parallel fluid flow problems, which relatively ease to have the transformation of non-linear and higher-order differentials using by discretized approximations with reasonable accuracy. A numerical method can also handle problems with a large number of variables and dimensions, which is difficult and time-consuming for the analytical methods. Over the past four decades, the solution of complex hydrodynamic and thermal instability problems has also been made possible by improved numerical techniques and continuously increasing computational capacity.

In practice, there are two alternatives to study the stability of steady flows. The first method consists of conducting a direct numerical simulation by solving the Navier-Stokes equations, thereafter starting near the steady state solution and verifying whether the corresponding time-dependent solution retracts to the steady state solution $[12,13]$. This type of computation is relatively expensive due to computational cost and time. The second approach employs the small perturbation method, wherein the flow variables are perturbed about the base state and the resulting partial differential equations are linearized. Subsequently, the linearized equations are used to formulate an eigenvalue problem whose solution dictates the stability of the base state.

Numerical assessment of stability is especially challenging for open boundary flows due to large domain requirements and necessitating the solution of an eigenvalue problem with high degrees of freedom. Although iterative methods exist, solution to such large eigenvalue problems is not trivial. Thus, alternative approaches for detection of bifurcation even for the simplest of flows need to be developed. In this work, we demonstrate a discrete, numerical form of linear stability analysis framework for prediction of onset of instability in two-dimensional laminar flows by using perturbed Navier-Stokes' equations in the vicinity of base flows which will be shown to be applicable for both open and confined domain flows. The degrees of freedom in the eigenvalue problem are reduced by developing the treatment in vorticity-stream function formulation. First we have validated this framework with a thermally driven flows by Winter [10] by developing the eigenvalue equations in the discrete sense and then demonstrate the applicability of the proposed method by calculating the critical parameters responsible for the onset of primary instability in a laminar flow past a square cylinder. A numerical solution of the resulting eigenvalue problem is also presented and shown to be in good agreement with published results.

\section{Methodology}

In this section, the formulation of the problem and the equations that govern the evolution of the disturbance are described. The numerical methods which are used to compute the base flow and formulation of eigenvalue problem for the determination of critical state are outlined here.

\subsection{Base flow computations}

The lattice Boltzmann Bhatnagar-Gross-Krook (BGK) two-dimensional nine velocities (D2Q9) model $[14,15]$ is used as the direct numerical solver for computing the base flow. The lattice Boltzmann method (LBM) is a simulation technique in which the discretized Boltzmann's equation is solved for the particle velocity distribution function $\left(f_{i}(\vec{x}, t)\right)$ on a regular, uniform Cartesian grid is given as,

$$
\frac{\partial f_{i}}{\partial t}+\mathbf{e}_{i} \cdot \nabla f_{i}=-\frac{\left(f_{i}-f_{i}^{e q}\right)}{\tau_{f}}+\phi_{i}
$$

Here $\tau_{f}$ is dimensionless relaxation time, $f_{i}$ is velocity distribution function, $f_{i}^{e q}$ is the equilibrium velocity distribution function (which depends on macroscopic properties such as density and velocity) and $\phi_{i}$ accounts for any external forces acting on the fluid is given as,

$$
\phi_{i}=w_{i}\left(1-\frac{1}{2 \tau_{f}}\right)\left\lfloor\frac{\mathbf{e}_{i}-\mathbf{u}}{c_{s}^{2}}+\frac{\mathbf{e}_{i}\left(\mathbf{e}_{i} \cdot \mathbf{u}\right)}{c_{s}^{4}}\right] \cdot \mathbf{F}
$$

The discretization of equation (2) in space and time leads to the lattice Boltzmann equation (LBE) which is given as,

$$
\begin{aligned}
& f_{i}\left(\mathbf{x}+\mathbf{e}_{i} \delta t, t+\delta t\right)-f_{i}(\mathbf{x}, t)= \\
& -\frac{\left(f_{i}(\mathbf{x}, t)-f_{i}^{\text {eq }}(\mathbf{x}, t)\right)}{\tau_{f}}+\phi_{i}
\end{aligned}
$$

The kinematic viscosity in the Navier-Stokes equation (derived from the above LBE) is

$$
v=\left(\tau_{f}-\frac{1}{2}\right) c_{s}^{2} \delta t
$$

where $c_{s}$ is the speed of sound and is related to lattice speed as, 


$$
c_{s}=\frac{1}{\sqrt{3}} c
$$

The equilibrium distribution functions are given as,

$$
f_{i}^{e q}=w_{i} \rho\left\lfloor 1+\frac{3}{c^{2}}\left(\mathbf{e}_{i} \cdot \mathbf{u}\right)+\frac{9}{2 c^{4}}\left(\mathbf{e}_{i} \cdot \mathbf{u}\right)^{2}-\frac{3}{2 c^{2}}(\mathbf{u} . \mathbf{u})\right\rfloor
$$

where $w_{i}$ is a weighing factor, $\mathbf{e}_{i}$ is the discrete velocity vector, and $c$ is the unit lattice speed given by $c=\delta x / \delta t$. This model is commonly known as the D2Q9 model as it is two-dimensional and involves nine velocity vectors. Figure 1 shows the lattice nodes of the D2Q9 model with nine velocities $\mathbf{e}_{i}$ defined by

$$
\mathbf{e}_{i}= \begin{cases}(0,0), & i=0 \\ ( \pm 1,0) c,(0, \pm 1) c, & i=1,2,3,4 \\ ( \pm 1, \pm 1) c, & i=5,6,7,8\end{cases}
$$

with

$$
w_{i}= \begin{cases}\frac{4}{9}, & i=0 \\ \frac{1}{9}, & i=1,2,3,4 \\ \frac{1}{36}, & i=5,6,7,8\end{cases}
$$

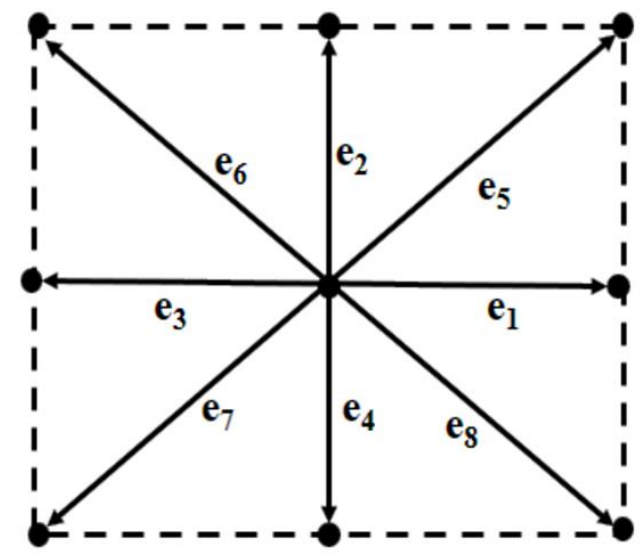

Figure 1. Illustration of lattice node of the D2Q9 model.

The pressure, density and velocity are computed from $f_{i}$ as,

$$
p / c_{s}^{2}=\rho=\sum_{i} f_{i} ; \quad \rho \mathbf{u}=\sum_{i} f_{i} \mathbf{e}_{i}
$$

For computing temperature-driven flows, the temperature distribution at different time instants are found by using the temperature lattice Boltzmann equation (TLBE). The TLBE is the set of temperature distribution function \{ $\left.g_{i}(x, t) \mid \alpha=0,1, \ldots \ldots . ., m\right\}$ from which the leading- order solution of the macroscopic temperature is obtained [16-20].

The evolution of the TLBE is written as,

$$
\begin{aligned}
& g_{i}\left(\mathbf{x}+\mathbf{e}_{i} \delta t, t+\delta t\right)-g_{i}(\mathbf{x}, t)= \\
& -\frac{\left(g_{i}(\mathbf{x}, t)-g_{i}^{\mathrm{eq}}(\mathbf{x}, t)\right)}{\tau_{g}}+s_{i}
\end{aligned}
$$

where $\tau_{g}$ is dimensionless relaxation time, $g_{i}$ is energy distribution function, $g_{i}^{e q}$ is the equilibrium energy distribution function (which depends on macroscopic properties such as temperature and velocity $\mathbf{u}$ ) and $s_{i}$ is a viscous heat dissipation term. The equilibrium distribution in equation (10) is written as

$$
g_{i}^{e q}=w_{i} T\left\lfloor 1+\frac{3}{c^{2}}\left(\mathbf{e}_{i} \cdot \mathbf{u}\right)\right\rfloor
$$

The temperature is computed from $g_{i}$ as,

$\rho \frac{1}{2} D_{o} R \mathbf{T}=\sum_{i} g_{i}$

The thermal diffusivity in the energy equation (derived from the above LBE) is given as,

$\chi=\frac{2}{5}\left(\tau_{g}-\frac{1}{2}\right) c_{s}^{2} \delta t$

The equations $(3,10)$ are conventionally solved in by two steps: (a) collision,

$$
\begin{aligned}
& \tilde{f}_{i}(\mathbf{x}, t)-f_{i}(\mathbf{x}, t)=-\frac{\left(f_{i}(\mathbf{x}, t)-f_{i}^{\mathrm{eq}}(\mathbf{x}, t)\right)}{\tau_{f}}+\phi_{i} \\
& g_{i}(\mathbf{x}, t)-g_{i}(\mathbf{x}, t)=-\frac{\left(g_{i}(\mathbf{x}, t)-g_{i}^{\mathrm{eq}}(\mathbf{x}, t)\right)}{\tau_{g}}
\end{aligned}
$$

and (b) streaming,

$$
\begin{array}{r}
f_{i}\left(\mathbf{x}+\mathbf{e}_{i} \delta t, t+\delta t\right)=\tilde{f}_{i}(\mathbf{x}, t) \\
g_{i}\left(\mathbf{x}+\mathbf{e}_{i} \delta t, t+\delta t\right)=g_{i}(\mathbf{x}, t)
\end{array}
$$

\subsection{Formulation of the eigenvalue problem}

The procedure for formulation of the discrete eigenvalue problem is described in this section. For illustration purposes, the equations governing flow past a square cylinder will be considered. For this situation, the non-dimensional form of the governing equations that describe the flow of an incompressible Newtonian fluid are,

$$
\begin{gathered}
\nabla \cdot \boldsymbol{u}=0 \\
\frac{\partial \boldsymbol{u}}{\partial t}+\boldsymbol{u} . \nabla \boldsymbol{u}=-\nabla p+\frac{1}{\operatorname{Re}} \nabla^{2} \boldsymbol{u}
\end{gathered}
$$

where $\boldsymbol{u}=(u, v)$ is the velocity vector and $p$ is pressure. In two-dimensions, the continuity and momentum equations (18) and (19) are transformed 
to vorticity $(\omega)$ transport and stream function $(\psi)$ form, given as

$$
\begin{gathered}
\frac{\partial \omega}{\partial t}+(\boldsymbol{u} . \nabla) \omega=\frac{1}{\operatorname{Re}} \nabla^{2} \omega \\
\nabla^{2} \psi=-\omega
\end{gathered}
$$

with $u=\frac{\partial \psi}{\partial y}, v=-\frac{\partial \psi}{\partial x}$ and vorticity $\omega=\frac{\partial v}{\partial x}-\frac{\partial u}{\partial y}$

. The introduction of $u=\frac{\partial \psi}{\partial y} \quad$ and $v=-\frac{\partial \psi}{\partial x}$ into equation (20) gives

$$
\frac{\partial \omega}{\partial t}+\frac{\partial \psi}{\partial y} \frac{\partial \omega}{\partial x}-\frac{\partial \psi}{\partial x} \frac{\partial \omega}{\partial y}=\frac{1}{R e} \nabla^{2} \omega
$$

To determine the flow stability, the flow is decomposed into a steady $(\bar{\omega}, \bar{\psi})$, usually called the base state or base flow, and an unsteady or perturbed part $\left(\omega^{\prime}, \psi^{\prime}\right)$ using

$$
\begin{aligned}
& \omega(x, y, t)=\bar{\omega}(x, y)+\varepsilon \omega^{\prime}(x, y, t), \\
& \psi(x, y, t)=\bar{\psi}(x, y)+\varepsilon \psi^{\prime}(x, y, t)
\end{aligned}
$$

where $x$ and $y$ are the space coordinate vectors and $\varepsilon \ll 1$ is a small number. Substituting into equations (21) and (22), subtracting the equations for the base flow and dropping higher-order terms the following linearized perturbation equation can be obtained for the perturbed vorticity and stream function,

$$
\begin{gathered}
\frac{\partial \omega^{\prime}}{\partial t}+\frac{\partial \bar{\psi}}{\partial y} \frac{\partial \omega^{\prime}}{\partial x}+\frac{\partial \psi^{\prime}}{\partial y} \frac{\partial \bar{\omega}}{\partial x}-\frac{\partial \bar{\psi}}{\partial x} \frac{\partial \omega^{\prime}}{\partial y}-\frac{\partial \psi^{\prime}}{\partial x} \frac{\partial \bar{\omega}}{\partial y} \\
=\frac{1}{R e} \nabla^{2} \omega^{\prime} \\
\nabla^{2} \psi^{\prime}=-\omega^{\prime}
\end{gathered}
$$

In this work, we represent the perturbed vorticity and stream function in terms of normal modes:

$$
\omega^{\prime}=\hat{\omega}(x, y) e^{-\sigma t}, \psi^{\prime}=\hat{\psi}(x, y) e^{-\sigma t}
$$

Here $\sigma$ is the complex growth rate. If $\sigma$ is real, the disturbances either grow or decay monotonically, with the critical Reynolds number to be that at which $\sigma=0$. If $\sigma$ is complex, then the neutral condition is when the real part of $\sigma=0$, and the onset of instability is oscillatory with a dimensionless frequency of the imaginary part of $\sigma$. Substituting (26) into (24) and (25), an eigenvalue problem with the growth rate being the eigenvalue can be formulated as

$$
\begin{aligned}
& \sigma \hat{\omega}=\frac{\partial \bar{\psi}}{\partial y} \frac{\partial \hat{\omega}}{\partial x}+\frac{\partial \hat{\psi}}{\partial y} \frac{\partial \bar{\omega}}{\partial x}-\frac{\partial \bar{\psi}}{\partial x} \frac{\partial \hat{\omega}}{\partial y}-\frac{\partial \hat{\psi}}{\partial x} \frac{\partial \bar{\omega}}{\partial y} \\
& -\frac{1}{\operatorname{Re}}\left(\frac{\partial^{2}}{\partial x^{2}}+\frac{\partial^{2}}{\partial y^{2}}\right) \hat{\omega}
\end{aligned}
$$

$$
\hat{\omega}+\left(\frac{\partial^{2}}{\partial x^{2}}+\frac{\partial^{2}}{\partial y^{2}}\right) \hat{\psi}=0
$$

For a structured and uniformly spaced grid as shown in Figure 2, the discretized form of equations (27) and (28) with second-order accuracy can be written as

$$
\begin{aligned}
& \sigma \hat{\omega}_{i, j}=\left(\frac{\bar{\psi}_{i, j-1}-\bar{\psi}_{i, j+1}}{2 \Delta y}\right)\left(\frac{\hat{\omega}_{i-1, j}-\hat{\omega}_{i+1, j}}{2 \Delta x}\right) \\
& +\left(\frac{\hat{\psi}_{i, j-1}-\hat{\psi}_{i, j+1}}{2 \Delta y}\right)\left(\frac{\hat{\omega}_{i-1, j}-\hat{\omega}_{i+1, j}}{2 \Delta x}\right) \\
& -\left(\frac{\bar{\psi}_{i-1, j}-\bar{\psi}_{i+1, j}}{2 \Delta x}\right)\left(\frac{\hat{\omega}_{i, j-1}-\hat{\omega}_{i, j+1}}{2 \Delta y}\right) \\
& -\left(\frac{\hat{\psi}_{i-1, j}-\hat{\psi}_{i+1, j}}{2 \Delta x}\right)\left(\frac{\hat{\omega}_{i, j-1}-\hat{\omega}_{i, j+1}}{2 \Delta y}\right) \\
& -\frac{1}{\operatorname{Re}}\left(\frac{\hat{\omega}_{i+1, j}-2 \hat{\omega}_{i, j}+\hat{\omega}_{i-1, j}}{\Delta x^{2}}\right) \\
& -\frac{1}{\operatorname{Re}}\left(\frac{\left.\hat{\omega}_{i, j+1}-2 \hat{\omega}_{i, j}+\hat{\omega}_{i, j-1}\right)}{\Delta y^{2}}\right) \\
& \left(\frac{\hat{\psi}_{i, j+1}-2 \hat{\psi}_{i, j}+\hat{\psi}_{i, j-1}}{\Delta y^{2}}\right)=0
\end{aligned}
$$

Equations (29) and (30) can be written in a condensed matrix form as

$$
\sigma\left(\begin{array}{ll}
1 & 0 \\
0 & 0
\end{array}\right)\left(\begin{array}{l}
\hat{\omega} \\
\hat{\psi}
\end{array}\right)=\left(\begin{array}{ll}
a_{11} & a_{12} \\
a_{21} & a_{22}
\end{array}\right)\left(\begin{array}{l}
\hat{\omega} \\
\hat{\psi}
\end{array}\right)
$$

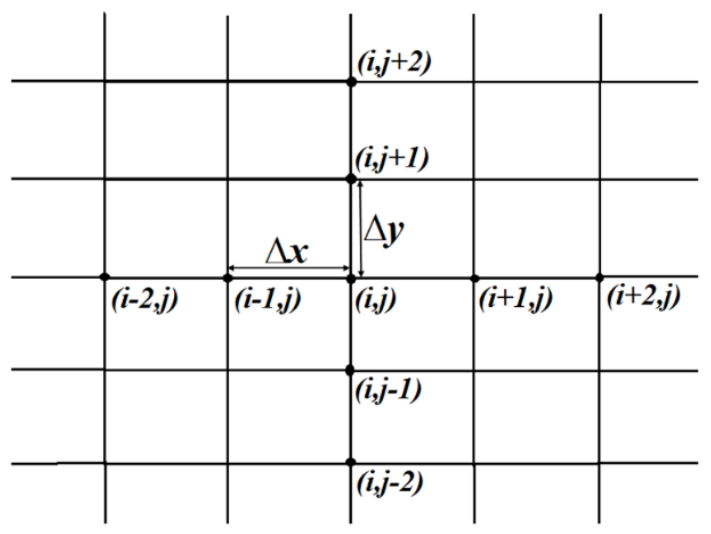

Figure 2. Typical grid spacing diagram and the nomenclature for the distances used. 
where coefficients $a_{11}, a_{12}, a_{21}, a_{22}$ are functions of the flow governing parameter associated with the base flow. The coefficients to these linear equations are determined by computing the base flow whose stability is to be established. The eigenvalue problem represented by equation (31) yields a generalized matrix eigenvalue problem of the form,

$$
J \boldsymbol{x}=\sigma M \boldsymbol{x}
$$

where $\boldsymbol{x}$ is an eigenvector that contains the unknown values of vorticity $\hat{\omega}$ and stream function $\hat{\psi}$. It can be seen from the differential equations that the mass matrix $M$ is singular, symmetrical and real, while the Jacobian matrix $\boldsymbol{J}$ is asymmetrical and real. This system of equations is solved by inverting the eigenvalue problem to a form that does away with the singularity of the mass matrix $M$. Thus, the system is written as

$$
\begin{gathered}
J^{-1} M x=\frac{1}{\sigma} x \\
B x=\eta x
\end{gathered}
$$

where $B=J^{-1} M$ and $\eta=1 / \sigma$

The size of the Jacobian and mass matrix depends on the number of perturbed parts $\left(\omega^{\prime}, \psi^{\prime}\right)$. For implementing and forming the discretized problem into eigenvalue problem of the type given by (34), the vector storage of perturbed space of solution is required. A variable is introduced that stores the global count of all grid points that are spanned in the discretized domain. The global counts are assigned sequentially, starting from one side of the boundary and traversing every point. This count variable maps the compass notation of the grid to vector storage location. In this way, the Jacobian matrix with defined locations for the constituting elements in the matrix is obtained by the pre-defined global count of the respective grid point. The method of assigning

\begin{tabular}{cccc}
\hline $\begin{array}{c}\text { Grid } \\
\text { point }\end{array}$ & $\begin{array}{c}\text { Global } \\
\text { count }\end{array}$ & $\begin{array}{c}\text { Storage location } \\
\text { for } x^{\prime}\end{array}$ & $\begin{array}{c}\text { Storage location } \\
\text { for } x^{\prime}\end{array}$ \\
\hline$i, j$ & $m$ & $2 m-1$ & $2 m$ \\
$i-1, j$ & $m_{1}$ & $2 m_{1}-1$ & $2 m_{1}$ \\
$i, j-1$ & $m_{2}$ & $2 m_{2}-1$ & $2 m_{2}$ \\
$i+1, j$ & $m_{3}$ & $2 m_{3}-1$ & $2 m_{3}$ \\
$i, j+1$ & $m_{4}$ & $2 m_{4}-1$ & $2 m_{4}$ \\
\hline
\end{tabular}

the Jacobian matrix for grid points shown in Fig. 2 is given in Table 1.

Table 1. Global count representation of all the points on the grid with appropriate neighbors.

To formulate the eigenvalue problem given by (34), the partial differential equations are discretized on a uniform grid using the finite-difference method.
Central differences are used for all the interior nodes, while forward or backward difference of second-order accuracy are used at the boundaries.

\subsection{Method of solution of the eigenvalue problem}

The critical eigenvalue is the leading value that crosses the imaginary axis as the control parameter increases. The instability of system (34) occurs when the real component of the critical eigenvalue crosses the imaginary axis from the right to the left half of the complex plane. Therefore, only the eigenvalues with the smallest real part are probed in this work.

When only one or few eigenvalues and eigenvectors are desired, the commonly used technique is the simultaneous iterative method. This method finds the eigenvalues with the smallest norm and the associated eigenvectors.

The simultaneous iterative method was developed by Bauer [21] and discussed in detail by Wilkinson [22]. The method described by Stewart and Jennings [23] captures the desired number of eigenvalues (say, $m$ ) with the smallest magnitude.

\section{Framework validation}

\subsection{Double-glazing problem}

The flow in a double-glazing or a two-dimensional air-filled rectangular cavity with differentially heated sidewalls and conducting horizontal surfaces along with Boussinesq approximation is an excellent test case for thermal instability since it is challenging to obtain accurate solutions for increasing Rayleigh numbers. Winters [10] studied the stability of this problem by computing the eigenvalues of the Jacobian matrix and mass matrix of the corresponding steady flow to determine the Hopf bifurcation point. Winters [10] concentrated on verification of the measurements published by Briggs and Jones [24], who had conducted similar experiments on the double-glazing problem by maintaining the vertical sidewalls at fixed temperatures. The results that Briggs and Jones [24] have published give the Rayleigh number regimes in which the flow is stable and also the critical Rayleigh number at which oscillatory behavior in the flow can be observed.

A pictorial representation of geometry and boundary conditions for double glazing problem is shown in Figure 3. For this case study, the nondimensionalization of Navier-Stokes and energy equations is accomplished by three scales [10]. These scales are the temperature scale $S_{T}$ which is 
the temperature difference between the hot and cold side walls, the length scale $S_{L}$ being the width of the cavity, and the velocity scale $\chi / S_{L}(\sqrt{R a})$ where $R a$ is Rayleigh number and $\chi$ is the thermal diffusivity. The non-dimensional form of continuity, momentum and energy equation is written as

$$
\begin{gathered}
\nabla \cdot \boldsymbol{u}=0 \\
\frac{\partial u}{\partial t}+u \frac{\partial u}{\partial x}+v \frac{\partial u}{\partial y}+\frac{\partial p}{\partial x}-\frac{\operatorname{Pr} \nabla^{2} u}{\sqrt{R a}}=0 \\
\frac{\partial v}{\partial t}+u \frac{\partial v}{\partial x}+v \frac{\partial v}{\partial y}+\frac{\partial p}{\partial y}-\frac{\operatorname{Pr} \nabla^{2} v}{\sqrt{R a}}=\operatorname{Pr} \theta \\
\frac{\partial \theta}{\partial t}+u \cdot \nabla \theta=\frac{1}{\sqrt{R a}} \nabla^{2} \theta
\end{gathered}
$$

The control parameters, namely the Prandtl number, $P r$, and the aspect ratio $(\gamma)$ are set to the values that have been used earlier, i.e., $\operatorname{Pr}=0.71$ and $\gamma=1.0$ [10]. The above equations contain two nondimensional groups, the Rayleigh number $R a=g \kappa S_{T} S_{L}^{3} / \chi v$ and Prandtl number $\operatorname{Pr}=v / \chi$, where $g$ is the acceleration due to gravity, $\kappa$ is the coefficient of volumetric expansion and $v$ is the kinematic viscosity.

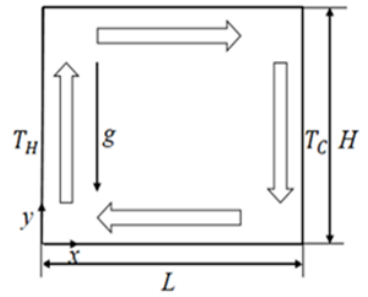

(a)

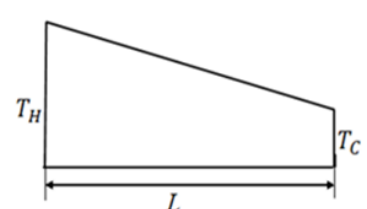

(b)
Figure 3. (a) Enclosure geometry and boundary conditions and (b) temperature boundary condition along the bottom and top walls of the square enclosure.

The base temperature distribution for such problem at a particular Rayleigh number and at different time instants is found by using the temperature lattice Boltzmann equation (TLBE). The grids for both the base flow and eigenvalue problems are the same and uniform.

The procedure for locating the Hopf bifurcation points is as follows. Steady solutions are obtained at Rayleigh numbers $1.0 \times 10^{6}, 1.5 \times 10^{6}$ and $2.0 \times 10^{6}$. Subsequently, at each of the Rayleigh numbers, the complex eigenvalue spectrum of the mass and Jacobian matrix is explored. It is found that one complex eigenvalue crosses the imaginary axis onto a left half plane at Rayleigh number of $2.0 \times 10^{6}$.
This indicates that one Hopf bifurcation from the steady solution occurs for Rayleigh number in the range $1.5 \times 10^{6}$ and $2.0 \times 10^{6}$. It is found that at $R a=1.87 \times 10^{6}$ the leading eigenvalues are on right half of imaginary axis and no unstable mode is present. At $R a=1.88 \times 10^{6}$ two complex conjugate unstable modes start to appear with the eigenvalue pair crossing the imaginary axis onto the left half plane.

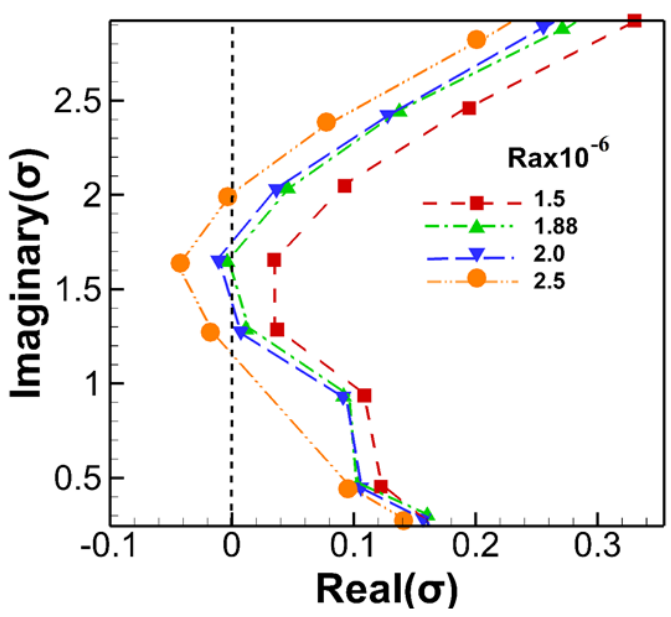

(a)

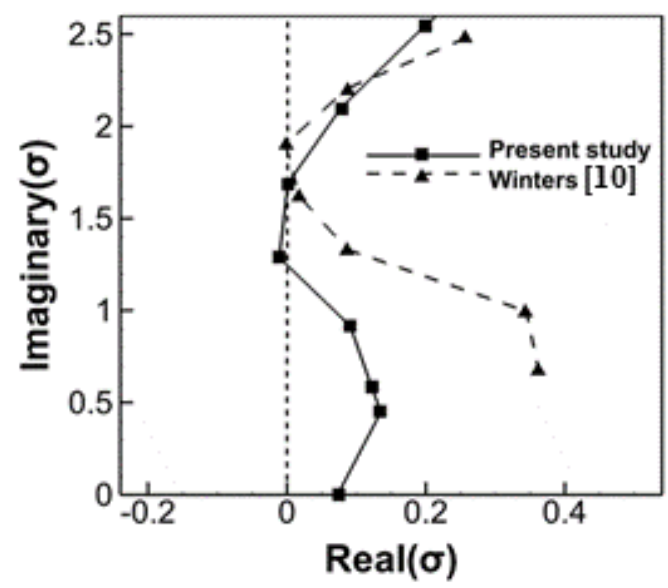

(b)

Figure 4. (a) Plots of the complex eigenvalues spectrum at four critical values of the Rayleigh number. Only eigenvalues with a positive imaginary part are shown. Dashed lines join eigenvalues computed at the same Rayleigh number (b) Comparison of results with Winters [10].

Figure 4(a) shows part of the eigenvalues spectrum at four Rayleigh numbers. Only the eigenvalues of the positive imaginary part are plotted. The crossing 
of the eigenvalue pair at $\operatorname{Real}(\sigma)=0$ represents a Hopf bifurcation and marks the onset of oscillations in the flow field of the double-glazing problem.

In this problem, the start of oscillations is predicted at a Rayleigh number of $1.88 \times 10^{6}$ which is in good agreement with the value $2.109 \times 10^{6}$ predicted by Winters [10]. The comparison of results with Winters [10] at Rayleigh number of $2.04 \times 10^{6}$ is shown in Figure 4(b).

The steady-state contours and eigenvectors (real and imaginary part) of stream function and isotherms at Rayleigh number of $1.87 \times 10^{6}$ are plotted in Figure 5.

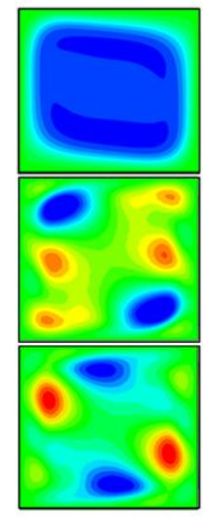

(a)

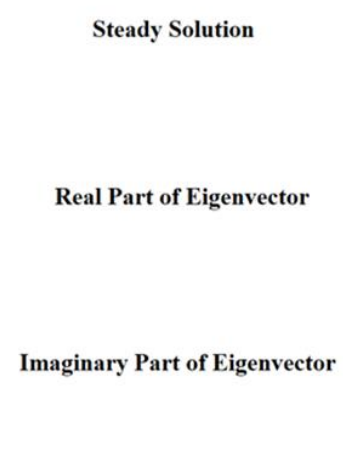

.

(b)
Figure 5. (a) Stream function and (b) isotherm twenty evenly spaced contours between -0.025 (blue) and +0.025 (red) for the steady-state solution and the real and imaginary parts of the eigenvector at critical Rayleigh number of $1.87 \times 10^{6}$.

On observing the spatial flow pattern arising near the vertical walls the presence of a fixed temperature, either high or low, which builds up the thermal boundary layer at the vertical walls can be easily noticed. It is also apparent that where the eigenvectors are much broader, the base temperature and momentum boundary layers are narrower. The eigenvector plots at Rayleigh number of have a reflectional symmetry about the vertical and horizontal axes which pass through the center of the cavity.

\section{Results and Discussion}

To demonstrate the applicability of the proposed discrete linear stability framework, we performed numerical simulations for a standard fluid flow problem as described below. In this case, LBM is used for base flow computation, the finite-difference formulation is used for formation of eigenvalue problem, and the simultaneous iteration method is used for the solution of the eigenvalue problem.

\subsection{Uniform flow past a square cylinder}

An analysis of uniform flow past a square cylinder is presented to capture the instability leading to shedding of vortices from the cylinder surface. The controlling parameter in this flow is Reynolds number, $R e=U_{\infty} a / v$ defined in terms of freestream velocity $U_{\infty}$, length of a side of the solid cylinder $a$ and the kinematic viscosity of the fluid $v$. At low Reynolds number $(R e<1)$, no separation takes place at bluff body surface because viscous forces dominate. Further, on increasing the Reynolds number, flow separation starts to appear with symmetrical vortex formation. The transition of vortex shedding from symmetric to asymmetric wake pattern occurs at a critical Reynolds number. When this critical Reynolds number is exceeded, the well-known Von Karman vortex street is formed with vortices shed periodically behind the cylinder.

The flow around bluff bodies has been studied by many groups who primarily focused on circular cylinder under free-flow conditions. However, there is a wide range of critical Reynolds number that has been reported in the literature. These critical values have been shown to depend on the blockage ratio, defined as the ratio of the cross-stream projection of the square (characteristic dimension of the cylinder) to the domain width. Kelkar and Patankar [25] determined $R e=53$ based on numerical linear stability analysis of the steady flow at a blockage of $14.2 \%$, while Davis et al. [26] found the critical value of Reynolds number experimentally within the range of $47 \pm 2$. Sohankar et al. [12] determined the critical value of Reynolds number as $51.2 \pm 1.0$ for a $5 \%$ blockage based on numerical simulations by using linearized Stuart-Landau equation and found that critical Reynolds number increases with increasing blockage.

In the present study, the focus is on the use of linear stability analysis to predict the critical Reynolds number for the onset of vortex shedding behind the square cylinder that will lead to unsteadiness in the flow and to locate the point of bifurcation. This is attempted by capturing the base flow at steady state and using it to determine its stability for various values of $R e$ using the method outlined in section 2.2. The layout of the computational domain used in this work along with the location of the fixed square cylinder and imposed boundary conditions is shown in Figure 6. Incompressible flow with constant fluid properties is considered. All geometrical lengths are 
scaled with $a$. The parameter which plays a dominant role in finding the critical value of Reynolds number is the blockage ratio of the domain, defined as $B=a / H$, where $H$ is the domain height. The blockage ratio in this work is fixed at $B=1 / 20$ or $5 \%$.

The procedure for locating the onset of vortex shedding is as follows. Steady solutions were obtained at Reynolds number of 45, 50 and 55 on a regular grid as fine as $700 \Delta x \times 400 \Delta y$. At each of the Reynolds numbers, the complex eigenvalue spectrum of the mass and sparse Jacobian matrices were explored by using simultaneous iteration method [23]. Figure 7 shows part of the eigenvalues spectrum at three different Reynolds numbers.

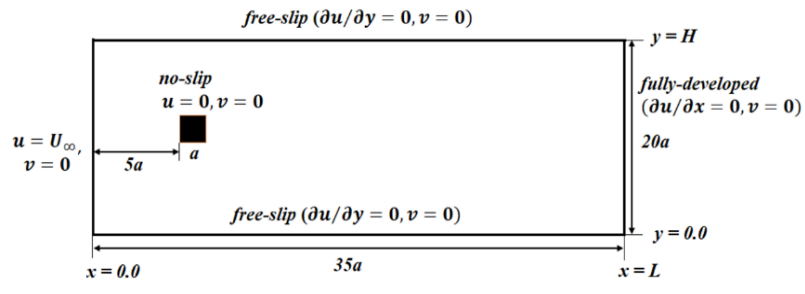

Figure 6. Layout of the computational domain with the imposed boundary conditions.

The crossing of the eigenvalue pair at $\operatorname{Real}(\sigma)=0$ represents a Hopf bifurcation and marks the onset of periodicity in the square cylinder wake. It was found that one complex conjugate eigenvalue pair crosses the imaginary axis onto a left half plane when Reynolds number reached 55. This behavior indicates that one Hopf bifurcation from the steady solution occurs for Reynolds numbers between 50 and 55 .

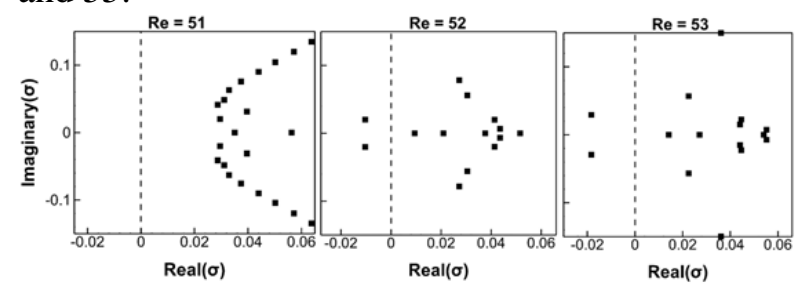

Figure 7. Distribution of smallest eigenvalues for normal mode, at $R e=51,52$ and 53.

Further, it is observed that all eigenvalues are on right half of imaginary axis at a Reynolds number of 51 and no unstable mode is present, while unstable patterns start to appear and one complex conjugate eigenvalue pair cross the imaginary axis onto the left half plane at Reynolds number of 52. To obtain a better understanding of solutions at different Reynolds numbers, the lift coefficient is selected as the raw signal and its instantaneous value at $R e=52$ is plotted in Figure 8. The abscissa is normalized with respect to the characteristic timescale $\tilde{t}=a / U_{\infty}$. The insets show flow patterns at different time instants of the simulation. Figure 8 shows that the instability in the flow resulting in the onset of periodic vortex shedding manifests due to round-off only after $\sim 400 \tilde{t}$. A more precise determination of the critical $R e$ requires a simulation for an even longer time duration which sometimes may not be even computationally feasible. On the other hand, the linear stability analysis method allows the use of a seemingly steady-state base flow to be used for determining its stability with excellent accuracy. The beginning of oscillations in this problem is determined at a Reynolds number of 52 which is very close to the results obtained by Sohankar et al. [12] and Kelkar and Patankar [25].

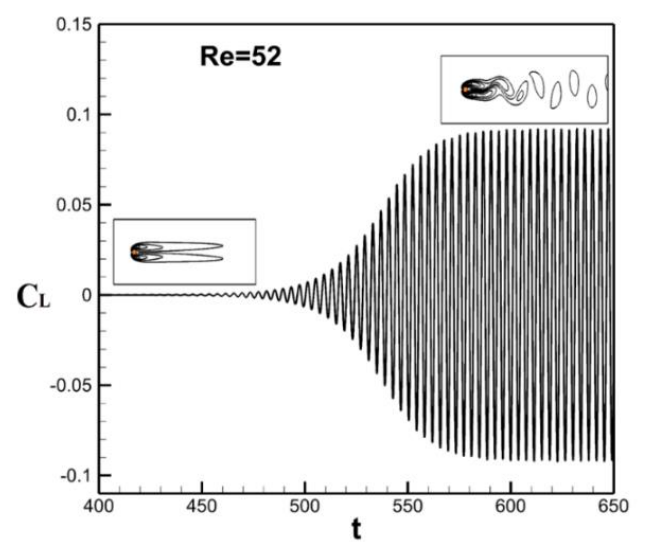

Figure 8 . The onset of vortex shedding at $R e=52$

The eigenvalue analysis also yields complex eigenvectors. The spatial form of the eigenvectors at the bifurcation point corresponding to $R e=51$ and critical point (i.e. $R e=52$ ) is shown in Figure 9 and 10 , respectively.

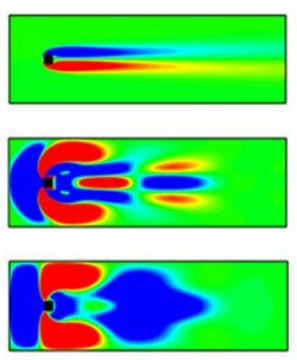

(a)

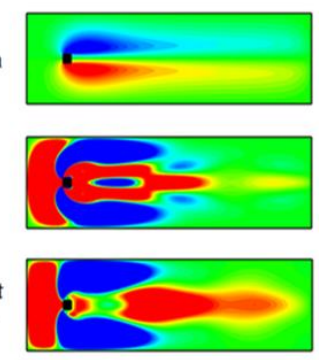

(b)
Figure 9. (a) vorticity and (b) stream function twenty evenly spaced contours between -0.60

(blue) and +0.60 (red) for the steady-state solution and the real and imaginary parts of the eigenvector at critical Reynolds number of 51. 
It is clear from Figure 9 that both vorticity and stream function eigenvectors for steady flow have reflectional symmetry about the horizontal axis. On the other hand, and as shown in Figure 10, this symmetry breaks for $R e=52$ due to the eigenvalue becomes negative.

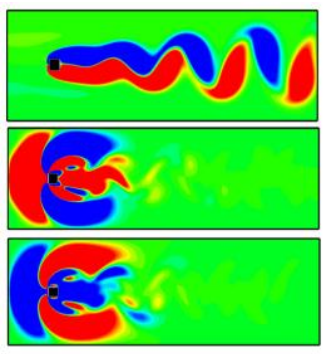

(a)

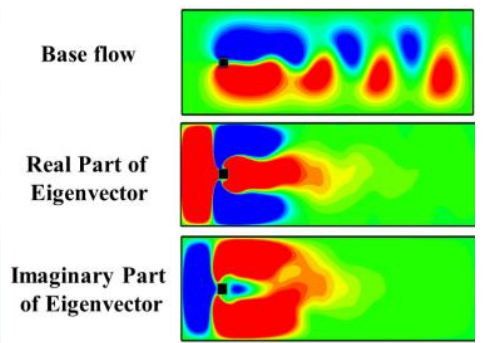

(b)
Figure 10. (a) vorticity and (b) stream function twenty evenly spaced contours between -0.60

(blue) and +0.60 (red) for the steady-state solution and the real and imaginary parts of the eigenvector at critical Reynolds number of 52.

\section{Conclusion}

This present work was directed towards formulation and development of a discrete linear stability analysis framework to numerically examine the onset of vortex sheading in the flow past a square cylinder. The two-dimensional base flow associated with the square cylinder at different Reynolds numbers are computed using the lattice Boltzmann method. The equations that govern the evolution of perturbations in the base flow constitute a generalized eigenvalue problem for the normal mode growth. The discrete version of this eigenvalue problem is first utilized to form the Jacobian and sparse mass matrices and then simultaneous iteration method is used for computing the eigenvalue spectrum. The results indicate that in the case of flow past a square cylinder the onset of two-dimensional vortex shedding is found at $R e=52$ which is in good agreement with earlier reported range $(47 \leq R e \leq 53)$. At $R e=51$ eigenvector patterns is symmetric about the $x$-axis, while they lose their symmetry at $R e=52$ due to eigenvalue becomes negative. In this study, the highest degree of freedom used is $1.6 \times 10^{5}$, which provides satisfactory results for two-dimension computation. Further improvement in the accuracy of locating the critical parameter requires an increase in the degrees of freedom that can be brought about by the parallelization of the present numerical analysis. The finite-difference method is ideally suited to linear stability analysis studies as it is easy to modify and to handle complex flows and geometries where the mathematics is not tractable. The framework that we have developed is sufficient to predict at the outset whether a hydrodynamic or a thermal system will unstable or not after some time, greatly reducing computational cost and time in making an accurate prediction. As presented, if the real part of the smallest eigenvalue is positive and the eigenvectors have a symmetry then there is no instability. On the other hand, the flow asymmetry transpired when the real part of the smallest eigenvalue pair crosses the imaginary axis onto the left half-plane, accompanied by the breaking down of corresponding eigenvector symmetry.

The flow past a square cylinder and double-glazing problems are only a few from the vast varieties of problems where the developed method may find applications. An interesting application of the developed framework is to predict the onset of instability due to the transition of vortex shedding from symmetric to asymmetric that leads to propulsion in a rigid or flexible wing plunging into a quiescent medium [27]. It is anticipated that this analysis will provide guidance for the onset of propulsion that can be attained for given controlling parameters and will be of significance in the design of systems with similar concepts. This will be the subject of a future publication.

\begin{tabular}{ll}
\multicolumn{2}{l}{ Nomenclature: } \\
$a$ & length of a side of the solid cylinder, [m] \\
$B$ & blockage ratio of the domain, [-] \\
$g$ & acceleration due to gravity, $\left[\mathrm{m} / \mathrm{s}^{2}\right]$ \\
$h$ & domain height, [m] \\
$\boldsymbol{J}$ & Jacobian matrix, [-] \\
$M$ & mass matrix, [-] \\
$p$ & Pressure, [N/m $\left.{ }^{2}\right]$ \\
$P r$ & Prandtl number \\
& $(P r=v / \chi),[-]$ \\
$R a$ & Rayleigh number \\
& $\left(=g \kappa S_{T} S_{L}^{3} / \chi v\right),[-]$ \\
$R e$ & Reynolds number $\left(=U_{\infty} a / v\right),[-]$ \\
$S_{L}$ & length scale, [m] \\
$S_{T}$ & temperature scale, $[\mathrm{K}]$ \\
$\tilde{t}$ & characteristic timescale \\
& $\left(=a / U_{\infty}\right),[\mathrm{s}]$ \\
$t$ & Time, [s] \\
$\boldsymbol{u}$ & the velocity vector of the fluid, $[\mathrm{m} / \mathrm{s}]$ \\
$U_{\infty}$ & free-stream velocity, [m/s] \\
$\boldsymbol{x}$ & Eigenvector, [-]
\end{tabular}

Nomenclature:

$a$ length of a side of the solid cylinder, [m]

$B$ blockage ratio of the domain, [-]

$g \quad$ acceleration due to gravity, $\left[\mathrm{m} / \mathrm{s}^{2}\right]$

$h$ domain height, [m]

$\boldsymbol{J}$ Jacobian matrix, $[-$

$M$ mass matrix, $[-]$

$p$ Pressure, $\left[\mathrm{N} / \mathrm{m}^{2}\right]$

$\mathrm{Pr}$ Prandtl number

$R a \quad$ Rayleigh number

Re Reynolds number $\left(=U_{\infty} a / v\right),[-]$

$S_{L} \quad$ length scale, $[\mathrm{m}]$

$S_{T} \quad$ temperature scale, $[\mathrm{K}]$

$\tilde{t} \quad$ characteristic timescale

$t$ Time, [s]

$\boldsymbol{u}$ the velocity vector of the fluid, $[\mathrm{m} / \mathrm{s}]$

$U_{\infty}$ free-stream velocity, $[\mathrm{m} / \mathrm{s}]$

- 
Greek letters

$\kappa \quad$ coefficient of volumetric expansion, $\left[\mathrm{K}^{-1}\right]$

$v \quad$ kinematic viscosity of the fluid, $\left[\mathrm{m}^{2} / \mathrm{s}\right]$

$\sigma \quad$ complex growth rate, [-]

$\chi \quad$ thermal diffusivity, $\left[\mathrm{m}^{2} / \mathrm{s}\right]$

$\psi \quad$ stream function, $\left[\mathrm{m}^{2} / \mathrm{s}\right]$

$\omega$ vorticity transport, $\left[\mathrm{s}^{-1}\right]$

\section{Acknowledgments:}

N.K. acknowledge the financial support received from Technical Education Quality Improvement Programme (TEQIP-III) of Uttarakhand Technical University, Dehradun. The authors also thank the IIT Delhi HPC facility for computational resources.

\section{References:}

[1]Tsai, B.-J. and Y.-C. Fu, Design and aerodynamic analysis of a flapping-wing micro aerial vehicle, Aerospace Science and Technology, 13 (2009), 7, pp. 383-392.

[2]Chandrasekhar, S., Hydrodynamic and hydromagnetic stability, Courier Corporation Inc., New York, USA, 2013.

[3]Drazin, P.G. and W.H. Reid, Hydrodynamic stability, Cambridge university press, Cambridge, UK, 2004.

[4]Jackson, C., A finite-element study of the onset of vortex shedding in flow past variously shaped bodies, Journal of fluid Mechanics, 182 (1987), pp. 23-45.

[5]Zebib, A., Stability of viscous flow past a circular cylinder, Journal of Engineering Mathematics, 21 (1987), 2, pp. 155-165.

[6]Kim, I. and A.J. Pearlstein, Stability of the flow past a sphere, Journal of Fluid Mechanics, 211 (1990), pp. 73-93.

[7]Fortin, A., M. Jardak, J. Gervais, and R. Pierre, Old and new results on the two-dimensional Poiseuille flow, Journal of Computational Physics, 115 (1994), 2, pp. 455-469.

[8]Clever, R. and F. Busse, Transition to timedependent convection, Journal of Fluid Mechanics, 65 (1974), 4, pp. 625-645.

[9]Busse, F., Non-linear properties of thermal convection, Reports on Progress in Physics, 41 (1978), 12, pp. 1929.

[10]Winters, K., Hopf bifurcation in the doubleglazing problem with conducting boundaries, Journal of heat transfer, 109 (1987), 4, pp. 894898.

[11]Xin, S. and P. Le Quéré, Linear stability analyses of natural convection flows in a differentially heated square cavity with conducting horizontal walls, Physics of Fluids, 13 (2001), 9, pp. 2529-2542.

[12]Sohankar, A., C. Norberg, and L. Davidson, Low-Reynolds-number flow around a square cylinder at incidence: study of blockage, onset of vortex shedding and outlet boundary condition, International journal for numerical methods in fluids, 26 (1998), 1, pp. 39-56.

[13]Sohankar, A., C. Norbergb, and L. Davidson, Numerical simulation of unsteady low-Reynolds number flow around rectangular cylinders at incidence, Journal of Wind Engineering and Industrial Aerodynamics, 69 (1997), pp. 189201.

[14]Arora, N., A. Gupta, S. Sanghi, H. Aono, and W. Shyy, Lift-drag and flow structures associated with the "clap and fling" motion, Physics of Fluids, 26 (2014), 7, pp. 071906.

[15]Succi, S., The lattice Boltzmann equation: for fluid dynamics and beyond, Oxford university press Inc., New York, USA, 2001.

[16]Guo, Z., B. Shi, and C. Zheng, A coupled lattice BGK model for the Boussinesq equations, International Journal for Numerical Methods in Fluids, 39 (2002), 4, pp. 325-342.

[17]He, X., S. Chen, and G.D. Doolen, A novel thermal model for the lattice Boltzmann method in incompressible limit, Journal of Computational Physics, 146 (1998), 1, pp. 282300.

[18]He, X. and L.-S. Luo, Theory of the lattice Boltzmann method: From the Boltzmann equation to the lattice Boltzmann equation, Physical Review E, 56 (1997), 6, pp. 6811.

[19]Li, L., R. Mei, and J.F. Klausner, Boundary conditions for thermal lattice Boltzmann equation method, Journal of Computational Physics, 237 (2013), pp. 366-395.

[20]Liu, C.-H., K.-H. Lin, H.-C. Mai, and C.-A. Lin, Thermal boundary conditions for thermal lattice Boltzmann simulations, Computers \& Mathematics with Applications, 59 (2010), 7, pp. 2178-2193.

[21]Bauer, F.L., Das verfahren der treppeniteration und verwandte verfahren zur lösung algebraischer eigenwertprobleme, Zeitschrift für angewandte Mathematik und Physik ZAMP, 8 (1957), 3, pp. 214-235.

[22]Wilkinson, J.H., The algebraic eigenvalue problem, Oxford Clarendon, 1965.

[23]Stewart, W.J. and A. Jennings, A simultaneous iteration algorithm for real matrices, $A C M$ Transactions on Mathematical Software (TOMS), 7 (1981), 2, pp. 184-198.

[24]Briggs, D. and D. Jones, Two-dimensional periodic natural convection in a rectangular enclosure of aspect ratio one, Journal of heat transfer, 107 (1985), 4, pp. 850-854. 
[25]Kelkar, K.M. and S.V. Patankar, Numerical prediction of vortex shedding behind a square cylinder, International Journal for Numerical Methods in Fluids, 14 (1992), 3, pp. 327-341.

[26]Davis, R., E. Moore, and L. Purtell, A numerical-experimental study of confined flow around rectangular cylinders, The Physics of Fluids, 27 (1984), 1, pp. 46-59.

[27]Vandenberghe, N., J. Zhang, and S. Childress, Symmetry breaking leads to forward flapping flight, Journal of Fluid Mechanics, 506 (2004), pp. 147-155.

\section{Creative Commons Attribution License 4.0} (Attribution 4.0 International, CC BY 4.0)

This article is published under the terms of the Creative Commons Attribution License 4.0

https://creativecommons.org/licenses/by/4.0/deed.en_US 